was given by Major Urwick, who drew freely on military organisation to illustrate his principles and points, while his series of diagrams and charts greatly enhanced the value of the paper. The main principle of organisation is that of co-ordination, and the task of leadership is largely that of adjusting and correlating continuously the various authorities and responsibilities which arise in any large organisation where the work of individuals must be divided not merely horizontally into different tasks, but perpendicularly into different levels of authority and responsibility. The psychological conception of 'the span of attention' has its administrative counterpart in 'the span of control' and neglect of the recognised administrative principle, that in practice no human brain should attempt to supervise directly more than five or at most six other individuals whose work is interrelated, creates insoluble problems in administration.

Specialisation is essential in business and industrial enterprise, and if it is to be effective, specialisation must be to some extent authoritative. This consideration complicates the work of co-ordination, and the compromise so far adopted in practice, known as the 'staff and line' system, often suffers from a lack of understanding and confused thinking about the relationships involved. Even the most authoritative students of business organisation frequently miss the significance of the military distinction between the specialist troops and services and the 'staff' proper. Failure to appreciate the proper functions of officials in a 'staff' or advisory capacity or to recognise the necessity for this third type of control distinct both from 'line' and from 'specialised authority' are the chief obstacles to more effective co-ordination in civil administration. Lack of this co-ordinating mechanism multiplies the number of committees, in itself expensive, but still more serious is the petrification of leadership caused by overloading of administrative work, including the loss of the personal touch. This last, which is commonly attributed to the size of modern enterprise, is essentially due to the absence of staff organisation.

Rapid growth in scientific knowledge has placed an unprecedented strain on man's power of organisation, and the effects of that strain are becoming apparent. As Herbert Spencer wrote: "socially as well as individually, organisation is indispensable to growth : beyond a certain point there cannot be further growth without further organisation."

A review of the prineipal types of trade associations and combinations was given by Mr. A. B. Blake. $\mathrm{He}$ discussed their status and inherent advantages and disadvantages in an attempt to estimate their significance in the new world order and the methods by which they might be ordered for the general advantage.

\title{
Cosmic Rays and Nuclear Physics
}

$\mathrm{P}^{\mathrm{R}}$ ROF. R. A. MILLIKAN, in an address delivered at the Chicago Century of Progress meeting of the American Association for the Advancement of Science, discussed some of the recent work on cosmic rays, interpreting it in the light of his well-known view that the primary rays are super-gamma rays, and that they may be produced in the synthesis of atomic nuclei (Science, Aug. 25).

Prof. Millikan's earlier work on the absorption of the cosmic rays in deep mountain lakes led him to regard the radiation as composed of three or more energy bands distinguished by their absorption coefficients of roughly $0 \cdot 35,0 \cdot 38,0 \cdot 04,0.02$ (per metre of water). The assignment of energies to these bands depended at the time on extrapolation of $\gamma$-ray absorption formulæ. Millikan now points out that the processes involved in the absorption of the rays are mainly nuclear, and that the extrapolation of formulæ dealing with absorption by extra-nuclear electrons is not legitimate.

The new data come from the observations of ionising particles of high energy made in Wilson chamber experiments by Anderson, Kunze, and Blackett and Occhialini. The particles observed are electrons and positive particles of electronic mass (positrons) and the curvature of their tracks in a magnetic field indicates that their energies lie mostly in the range of $60-3,000$ million volts. Millikan considers that they are secondary rays produced by the absorption of photons, and this view is supported by the fact that Anderson and Neddermeyer have observed the production of photons by $\gamma$-rays from thorium $C^{\prime \prime}$. The hard thorium $C^{\prime \prime} \gamma$-rays have an energy of $2 \cdot 6$ million volts and when they pass into lead, paired positrons and electrons are frequently liberated. In many cases, the total energy of the pair is about 1 million volts less than the energy of the primary $\gamma$-rays. Following Dirac, it is suggested that the whole of the energy of a primary quantum is absorbed in a nuclear encounter of this type, and that the million volts energy deficiency appears as the mass of the two particles. Even apart from this theory, the experiments possibly show that the energy of the pair of particles may approach that of the primary rays.

Now Anderson finds that the positive and negative particles are about equally frequent in the photographs of cosmic ray tracks, and Millikan thinks that in this case, too, the particles are produced in pairs by the absorption of photons and that the energies of the pairs correspond closely with that of the primaries. In this case, about 30 per cent of the rays at sea-level lie in the range below 350 million volts. The effect of lead screens on the ionisation in an electroscope is consistent with this distribution of the energies of the ionising particles, and with a higher proportion of low energy particles at high altitudes. These absorption measurements do not, of course, throw new light on the relation between primary rays and ionising particles.

The energies thus ascribed to the cosmic rays correspond in order of magnitude with those that would be liberated by the synthesis of moderately light atoms from hydrogen nuclei with the disappearance of mass corresponding to the atomic weight data.

There are, of course, other difficulties in ascribing the origin of the cosmic rays to a multi-body condensation. Prof. Millikan, moreover, does not account for the coincidences observed by Rossi and others when two counters are separated by a heavy lead screen. It would require an electron of 3,000 million volts to penetrate the screen in one of Rossi's experiments, and such particles are very infrequent in Anderson's photographs. It is difficult to escape the conclusion that if the primary rays are photons, they may produce secondary particles at widely separated points. 